TITLE PAGE

Article title: Reconceiving Egg Freezing: Insights from an analysis of five years of data from a UK clinic

Authors: Zeynep B. Gürtin*, Trina Shah, Jinjun Wang, and Kamal Ahuja

* Corresponding Author

Zeynep B. Gürtin*

London Women's Clinic

113-115 Harley Street

London W1PG 6AP, UK

zbg20@cam.ac.uk

Trina Shah

London Women's Clinic

113-115 Harley Street

London W1PG 6AP, UK

Jinjun Wang

London Women's Clinic

113-115 Harley Street

London W1PG 6AP, UK

Kamal K. Ahuja

London Women's Clinic

113-115 Harley Street

London W1PG 6AP, UK 


\title{
Reconceiving Egg Freezing: Insights from an analysis of five years of data from a UK clinic
}

\begin{abstract}
Research Question: What can we learn from five years of egg freezing practice in the UK? What are patients' social and demographic characteristics, reasons for freezing their eggs, and decisions regarding subsequent storage or thawing?

Design: A retrospective analysis of clinical and laboratory data of all 514 cycles of 'own' egg freezing conducted at the London Women's Clinic in the five-year period from the start of 2012 to the end of 2016.

Results: This analysis, the first of its kind, develops a clearer picture of egg freezing trends in the UK and fills in the details behind the HFEA's national figures. We provide the reason for freezing for each of the 514 cycles by 352 patients, and identify four different categories of own egg freezing. To the established categories of 'medical' and 'social' already discussed in the literature, we add the two new categories of 'clinical' and 'incidental' egg freezing. We show how each of these categories presents a distinct egg freezing patient profile, and discuss the similarities and differences between them across variables such as age, relationship status, number of eggs frozen, number of cycles undertaken, and the current status of frozen eggs.

Conclusions: The data require us to reconceptualise the phenomenon of egg freezing, and argue for the importance of clearly and accurately differentiating between the categories in clinical and national data collection to adequately inform future practice, regulation and the decision-making processes of patients considering these procedures.
\end{abstract}

\section{INTRODUCTION}

Egg freezing, or oocyte vitrification, is one of the fastest growing assisted reproductive technologies (ARTs), attracting attention not only from potential users but more widely from social science scholars, ethicists, and the popular media. While the US is certainly leading this trend, with recent newspaper reports claiming that more than 20,000 American women have already elected to freeze their eggs (Belluck, 2018), egg freezing is also showing rapid growth in the UK, Israel, Australia, Scandinavia, and across many European countries, with some predicting its development into a big business on a global scale in the very near future (see Browne, 2018). Yet, as it is a relatively new technology, there is currently limited data available to understand the phenomenon and its characteristics, and little empirical evidence to support the range of views that have been expressed regarding the ethical aspects and social impact of such a technology (Baldwin et al., 2014).

The world's first birth from a frozen egg was reported in 1986 (Chen, 1986); however, due to the difficulties with successfully thawing eggs, and the subsequent low rates of fertilization and pregnancy, the technology remained marginal for the following two decades. It was the advent of vitrification, a method of flash freezing using liquid nitrogen, which reduced the potential damage to the internal structures of eggs, and thereby enabled success rates for thawing and fertilization of frozen eggs to improve (Argyle et al., 2016), that fuelled the growth of the practice. Following a 
series of randomized controlled trials (Cobo et al., 2008, 2010; Rienzi et al., 2010; Parmegiani et al., 2011), which suggested that in vitro fertilisation (IVF) using frozen-thawed eggs yielded comparable fertilization and pregnancy results as IVF cycles using fresh eggs, the American Society of Reproductive Medicine (ASRM) lifted the "experimental" label from the practice of egg freezing in 2012 (ASRM, 2013). This much cited announcement by the ASRM, as well as the recommendations from the ESHRE Task Force on Ethics and Law (Dondorp et al., 2012), helped both to develop the confidence of potential patients in cryopreservation and also to encourage the spread of the practice to many clinics.

In the UK, The Human Fertilisation and Embryology Authority (HFEA), the statutory body that regulates and inspects all clinics providing ARTs, has allowed the use of frozen eggs in fertility treatments since 2000 (Wise, 2000); although before 2007 clinics were almost exclusively practicing the old 'slow freezing' method, most have now fully transitioned to the use of vitrification. Despite years of regulated practice however, it was only in 2016, in 'Fertility Treatment 2014 - Trends and Figures' (HFEA, 2016), that the HFEA began to publish national data on egg freezing. Explaining the decision to include egg freezing for the first time in their report, Sally Cheshire, the Chair of the HFEA, stated in her foreword that, "Freezing techniques are relatively new, and were very rare only five or six years ago. Interest from patients and the media has grown considerably in recent years, however, and we felt it was time to provide more clarity." (HFEA, 2016: 2)

The HFEA's 2016 report provided a range of data on egg freezing in the UK, including the numbers of women freezing their eggs, the number of egg freezing cycles undertaken, the age of freezers, number of thaw cycles undertaken, how long the eggs had been frozen for, and the outcomes and success rates of thawed cycles, as well as the number of clinics providing egg freezing in the UK. The HFEA's latest report, released in March 2018 (HFEA, 2018), updates this data by providing further figures for egg freezing practice between 2014-2016. Although the two reports contain some important discrepancies due to the use of different definitions regarding what counts as an egg freezing cycle (see Gurtin, 2018), the HFEA data remain the most comprehensive data on egg freezing in the UK, and are commonly referenced in public and media debates, whether in support or criticism of the practice (e.g. Specter, 2017; Wooler, 2017).

However, it is important to note that the HFEA data leaves some significant questions about the practice and social context of egg freezing in the UK unanswered. For example, in the 2016 report, it is noted that only very limited information has been collected regarding women's reasons for seeking egg freezing. Instead, reasons are extrapolated from the available information, such as the fact that $54.1 \%$ of women aged 38 and older and $36.4 \%$ of women aged 37 and younger who froze their eggs recorded that they had 'no male partner'. The 2018 report provides no details at all for the reason of egg freezing. This makes it very difficult to gain an accurate picture of who is freezing their eggs and why they may be doing so. The 2016 report also points to some interesting questions regarding which patients are thawing their eggs, but stops short of providing answers. For example, 
the data show that, "Of the thawed egg cycles performed, well over half used eggs which had been frozen for less than a year. These are unlikely to be those women freezing in order to preserve their fertility in the longer term, but may be those that had to put their treatment on hold for unexpected reasons, for instance, if donor or partner sperm was not available at the right time." (HFEA, 2016:

27)

Taking the gaps in the HFEA data as our starting point, we wanted to shed light on some of the questions surrounding the social context of egg freezing in the UK; in particular, we wanted to develop a more accurate understanding of the different reasons why women had frozen their eggs, and how this related to other characteristics, such as their age or relationship status. We were also keen to find out whether our clinical experience could tell us something about why, according to national data, the majority of thawed eggs had been stored for less than a year, and the reasons behind such seemingly short storage periods. In this paper we report on the findings from a detailed analysis of five years of egg freezing at the London Women's Clinic Harley Street (LWC), from 2012 to end of 2016, based on patient and laboratory records.

\section{MATERIALS AND METHODS}

Data for this paper were gathered using a retrospective evaluation of the LWC's existing documentation regarding past patients. Patient records and laboratory data were used to create a comprehensive database of all own egg freezing cycles undertaken at the LWC.

First, all treatment cycles logged as 'own egg freezing' at the LWC were compiled in a spreadsheet. Then, the entries were double-checked manually to ensure that all were instances of own egg freezing, and any cycles of eggs frozen for donation were excluded. This left us with 521 cycles of own egg freezing from 2008 onwards, however we limited the current analysis to a five-year period, from 1 January 2012 to 31 December 2016, since 2012 was the first year in which there were more than five cycles of own egg freezing recorded. In all of the cycles, eggs were frozen using the vitrification method as the LWC laboratories did not use the 'slow freezing' method. This study sample thus represents 514 cycles of egg freezing undertaken by 342 women for their own later use, i.e. all 'own egg freezing cycles' undertaken at the LWC over the five-year period, from 2012 to end of 2016. (For the rest of this paper, for convenience "egg freezing" refers exclusively to "own egg freezing" and excludes any egg freezing as part of egg donation cycles or programmes.)

Researchers cross-checked each own egg freezing cycle logged against the relevant patient's records and lab sheets to create an excel spreadsheet which included the following details for all 514 cycles: year of cycle; month of cycle; date of egg collection; patient ID; patient age (at egg freeze); patient birth date; patient sexuality and/or relationship status (single, heterosexual couple, lesbian couple); egg freezing cycle number (for patient); number of eggs collected; number of eggs frozen; reason for 
egg freezing; current status of eggs (in storage, thawed for conception attempt, discarded, moved); and (if applicable) details and outcome of egg thaw.

Once the above data were recorded, they were examined, analysed for patterns and relevant categories were generated, regarding, for example, the reason for egg freezing. An in-depth numerical analysis was undertaken on various aspects of the data, including comparisons of different variables (such as age, number of eggs frozen, and current status of frozen eggs) between women who had frozen their eggs for different reasons. Statistical analyses, using the Chi-squared test and the one-way Anova, were applied as appropriate, in particular to determine the statistical significance of the different characteristics between patients freezing their eggs for different reasons. This retrospective analysis did not require ethical or institutional review board approval, as it assessed laboratory and clinical records from previously validated and approved procedures, practised under license from the HFEA.

\section{RESULTS}

As can be seen in Table 1, 6 women each underwent 1 cycle of egg freezing at the LWC in 2012. This increased to 52 cycles (undertaken by 43 women) in 2013; 91 cycles (by 71 women) in 2014; 179 cycles (by 135 women) in 2015; and 186 cycles by 137 women in 2016. This represents a dramatic increase in the number of egg freezing cycles at the LWC over the five years, with numbers more than doubling, and rising by $104.4 \%$, in the latest two years alone (see also Figure 1). Although our sample included a total of 342 women who froze their eggs in the five-year period, the total for each year added together gives a different figure (392). This is because 46 women froze their eggs in multiple cycles across two or three different years.

Looking at the overall patient group, we can see that $76.9 \%$ of patients were single at the time of egg freezing, $21.9 \%$ were in a heterosexual relationship, and $1.2 \%$ were in a same-sex relationship. (see Figure 2).

When we looked at patterns across the sample, we found that there were differences in characteristics such as average age and relationship status among egg freezing patients. These differences were associated with the reasons why women were freezing their eggs. Thus, in order to better understand how the freezing motivations effect other variables, we categorised egg freezing cycles according to the reason for egg freezing. This showed us that, within the LWC, there were four distinct categories of reasons for egg freezing: Social Egg Freezing (SEF), Medical Egg Freezing (MEF), Clinical Egg Freezing (CEF) and Incidental Egg Freezing (IEF). While the first two categories, SEF and $M E F$, are already established in the literature and public debates surrounding egg freezing, the third and fourth categories, CEF and IEF, are new categories we propose based on our detailed data analysis. We describe each of these categories in detail below. 


\section{Defining the categories}

Social Egg Freezing (SEF) is the largest of the four categories and is the one most commonly associated with the phenomenon of egg freezing. It refers to women who have chosen to freeze their eggs for so-called 'social reasons'. These women are predominantly concerned with natural age-related fertility decline, and opt for egg freezing as a means to increase their chances of motherhood in the future. (It is worth noting that we included three patients in this category who had frozen their eggs as part of a Freeze and Share scheme, in which they donated half of their eggs to another woman and in return were able to freeze the remaining half of their eggs for free or highly reduced cost.)

Medical Egg Freezing (MEF) refers to eggs frozen for medical reasons, including (in our sample) both eggs frozen prior to gender reassignment and prior to potentially fertility damaging medical treatments, such as chemotherapy for cancer. The number of MEF cycles in our sample was very small, hence it was not possible to determine whether there might be any significant intra-group differences. (For clinics or institutions performing large numbers of MEF cycles, it would be useful to conduct a further analysis to establish whether patients freezing eggs for different types of 'medical' reasons present different egg freezing profiles and trajectories.)

Clinical Egg Freezing (CEF) refers to cycles in which eggs were frozen for 'clinical' reasons, as an intentional part of the IVF treatment of certain patients. These patients were currently attempting conception through IVF, but had been advised to undergo egg freezing to 'batch' eggs, either because they were expected to produce a low number of eggs per cycle, or because they had a high likelihood of producing eggs with chromosomal abnormalities. The plan for these patients was to 'batch' eggs from multiple consecutive cycles of ovarian stimulation together, fertilize them in one go, and usually to perform preimplantation genetic testing, so that a healthy embryo (if available) could be transferred. As such, these patients were not using egg freezing to postpone conception per se, but to increase their chances of a healthy pregnancy through IVF. Although CEF was the second largest category within our sample, it is not clear to what extent such treatment protocols are used across different clinics.

And finally, Incidental Egg Freezing (IEF) refers to the 'incidental' freezing of eggs during what was intended to be a routine IVF cycle, when, for some reason, there was no sperm to enable fertilization on the day of egg collection. In our sample this included both patients whose male partners were unable to reach the clinic that day, as well as patients whose partner's semen sample proved unexpectedly - unusable on the day. In these cases, the eggs were frozen (as opposed to discarded) with the intention to fertilize them as and when a viable sperm sample could be provided.

\section{Number of egg freezing cycles and patients, by year and category}


Table 2 presents the number of egg freezing cycles and the number of women freezing their eggs, according to category of egg freezing for each year. We can see that while the large majority of egg freezing cycles across the five-year period refer to SEF (75.7\%), CEF (17.9\%) forms the second largest category; both IEF (4.3\%) and MEF (2.1\%) are much smaller categories. Figure 3 shows the relative size of each of the categories.

\section{Numbers of egg freezing cycles undertaken, average numbers of eggs frozen per cycle, and average age per cycle for each category}

Of the 342 egg freezers in our sample, 234 women (68.4\%) undertook one cycle of egg freezing only, $58(17.0 \%)$ undertook two cycles, and $50(14.6 \%)$ undertook three or more cycles (which includes 44 women who undertook three; three who undertook four; two who undertook six; and one woman who completed eight consecutive cycles of egg freezing).

As shown in Table 3, all women in the IEF category only undertook one cycle of egg freezing, whereas approximately a third of patients in the SEF category and approximately two-thirds of the patients in the CEF category had undertaken more than one cycle. Comparing SEF and CEF, the two largest categories, we found that while $70.5 \%$ of social freezers underwent only one cycle, $14.9 \%$ underwent two, and 14.6\% underwent three or more cycles of egg freezing; for CEF patients the respective percentages were $33.3 \%, 40.5 \%$ and $26.3 \%$. These differences were statistically significant $\left(X^{2}=23.6, p<.0001\right)$, reflecting that CEF patients were likely to undergo a higher number of egg freezing cycles.

Table 4 provides information on the average number of eggs frozen per cycle and the average age of the patient per cycle across the four different categories of egg freezing. (In each of these cases, the mean is taken as the average.) The differences between these categories were statistically significant as determined using a One-way Anova test (the f-ratio values are provided in Table $4, p<.0001$ ). According to these results MEF patients were the youngest, with an average age of 27.3 years, and CEF patients were the oldest, with an average age of 39.2. While the average age of all egg freezing patients is 37.5 , this obscures the average age differences between different groups, which may be important to consider with regards to the future outcomes for these patients, including potential success rates for fertilization and live birth, since they will be using eggs frozen at different ages.

Table 4 also shows that, as would be expected, the average age of patients in the different freezing categories was associated with the average number of eggs frozen per cycle; with the youngest patients in the MEF category freezing the highest average number of eggs (11.4), and the oldest patients in the CEF category freezing the lowest (4.7 eggs per cycle). It is interesting to note that while the mode number of eggs frozen per cycle by SEF patients is between 4 and 5 , the mean is 6.9, due to the wide distribution of this category, with a range from 0 to 28 eggs frozen in one cycle. 


\section{Age distribution of egg freezing patients in each category}

Looking across the 514 cycles, there is a wide age range of egg freezing patients from 22 to 46 years, with 37.5 years as the average age for all cycles. As Table 5 shows, over half of the egg freezing cycles $(52.4 \%$ ) in our sample were undertaken by women aged $35-39$; with $17.3 \%$ of cycles undertaken by women under 35 , and $30.4 \%$ by women aged 40 and over. Table 5 provides a breakdown by age group and category of freezing, while Figure 4 shows a bar chart representing the differential distribution of egg freezing cycles from different categories across the age groups. For example, while the highest number of SEF cycles are in the 35-37 year age group, the highest number of CEF cycles are undertaken by the 40-42 age group, and highest number of MEF cycles by the under 30 group.

\section{Relationship status of egg freezers by category}

Table 6 shows the relationship status of egg freezers in the different categories, with some striking differences between groups. For example, the two largest categories, SEF and CEF, show opposite characteristics: while $95.4 \%$ of SEF patients are single, this is only true for $1.1 \%$ of CEF patients. Conversely, while $98.9 \%$ of CEF patients are in a relationship, this is only true for $4.7 \%$ of SEF patients. These are highly statistically significant differences between the two categories $\left(X^{2}=\right.$ $388.73, p<.0001)$.

Thus, while the overall percentages of egg freezing patients who are single, in a heterosexual relationship or in a lesbian relationship, as shown by Figure 2 , correctly demonstrates the values for the sample as a whole, it fails to provide an accurate portrayal of any of the individual categories and obscures the distinct differences between them.

\section{Current status of frozen eggs}

As part of our analysis, we looked at the current status of the eggs that were frozen in each of the 514 cycles, and recorded whether they were still in storage (75.1\%); had been thawed to attempt conception (22.2\%); moved to another clinic (1.8\%); or discarded (1.0\%). These data are presented in Figure 6. As expected, the majority of eggs frozen across the five-year period 2012-2016 were still in storage at the time of analysis (Autumn 2017). However, looking comparatively at the constitution of eggs that have been thawed versus the eggs that are still in storage, some striking differences emerge regarding the relationships between the current status of eggs and the category of egg freezing. As can be seen in the Figure 6, while most of the eggs in storage (93.5\%) come from SEF cycles, the great majority of thawed eggs (85.1\%) come from CEF and IEF cycles.

For example, SEF cycles accounted for $75.7 \%$ of the 514 freezing cycles across five years, but only for $14.9 \%$ of the cycles in which eggs had been thawed. This is because eggs from $92.8 \%$ of the SEF cycles were still in storage, and only eggs from $4.4 \%$ of SEF cycles had been thawed to attempt conception (see Table 7). 
Conversely, while CEF cycles accounted for $17.9 \%$ of the 514 freezing cycles, this category accounted for a very high $72.8 \%$ of those in which eggs had been thawed. This is because eggs from $90.2 \%$ of CEF cycles had already been thawed, and only $7.6 \%$ were still in storage. The difference between these categories regarding the current status of frozen eggs was highly statistically significant $\left(X^{2}=\right.$ $332.9, p<.0001)$.

\section{DISCUSSION}

The current retrospective analysis responds to calls for more empirical enquiry into patients electing to freeze their eggs and monitoring of such practices (e.g. Argyle et al., 2016; Balwdin et al., 2014). Our results show the phenomenon of egg freezing to be more heterogeneous than hitherto assumed and enable us to develop a more accurate understanding of the various sub-groups of egg freezing patients. Contrary to assumptions to date, SEF, the category that has understandably captured most interest in recent years, is not the only category of egg freezing. Indeed, while SEF makes up the majority of egg freezing cycles in this sample, and also in the UK and across the globe more generally), it is extremely important to note the other three categories identified in this paper - CEF, IEF, and MEF - if we are to appreciate the full scope of oocyte vitrification practice and patterns. These different categories, while not exhaustive, show that oocyte vitrification is being used by different patient groups with different needs and desires, and it is crucial to recognise these differences if we are not to arrive at misleading conclusions. Based on the results presented in this paper, we have identified the following four distinct 'typical profiles' of egg freezing patients from each of the four categories, which clearly illustrate not only the demographic differences, but also the different clinical outcomes between patients from different groups.

\section{Typical profiles in each category}

Profile of SEF patient: The typical patient who has frozen her eggs for "social" reasons, as profiled from this data set, is 37.4 years old and single. She will most likely undertake only one cycle of egg freezing, perhaps two or three, freezing approximately 7 eggs in each cycle. She is very likely to still have her eggs in storage (even if she froze up to 5 years ago). This profile is similar to previously published demographic details of social egg freezers in the UK (Baldwin et al., 2015), from a study of 23 women, though it is worth noting that the women in our sample were a little older and more of them were single.

Profile of MEF patient: There are two predominant groups of medical egg freezers: women freezing before cancer treatment, and transgender patients preserving their fertility prior to gender transition. These patients also tend to be single, but they are significantly younger than social egg freezers, with an average age of 27.3 at the time of freezing. Most medical egg freezers undertake one cycle of egg freezing, with approximately 11 eggs frozen in that cycle. This group has the highest 
number of eggs frozen per cycle, on account of their younger age. The eggs are likely to stay in storage for a long time.

Profile of CEF patient: Clinical egg freezing refers to all cycles where the freezing of eggs was clinically intended, usually in order to maximise the chances of a pregnancy in cases with a poor prognosis. As a group, these women are the oldest, with an average age of 39.2 years, and have 4.7 eggs (the lowest average) collected per cycle. These characteristics make sense with regard to the treatment protocol of this population, since the justification for clinically freezing eggs is to "batch" the small number of eggs collected from multiple cycles in order to fertilize them all at once, often with subsequent genetic testing of the embryos, before implanting one healthy embryo (if available) back to the uterus. The typical patient whose eggs are frozen for clinical reasons is in a heterosexual relationship, and will undertake two or more cycles. The rates of egg thaw in this group are very high, since the goal of the treatment is to achieve a pregnancy, and not to keep eggs stored for any length of time.

Profile of IEF patient: A patient whose eggs are frozen for incidental reasons would have been going through a standard IVF cycle but had to have her eggs frozen for an unexpected reason, usually because there was no sperm available on the day of egg collection. This may be because the partner could not arrive at the clinic for some reason, because he was unable to produce a sample, or because, unexpectedly, the semen sample produced on the day contained no sperm cells. The women in this group are all in heterosexual relationships, aged on average 35.5 years old, and freeze approximately 9 eggs. These women only undergo one cycle of freezing, since even this cycle was unintentional and necessitated by unforeseen circumstances. There is a high rate of thaw, although thawing of eggs may be delayed if the partner needs treatment, or may even be abandoned altogether if sperm cells cannot be found and the couple is unwilling to use donor sperm.

\section{Using our analysis to shed light on national trends and figures}

Looking at the last two HFEA reports $(2016,2018)$, we can see that our data shed light on some of the most pressing questions posed by the national figures, and highlight some of the realities of egg freezing unintentionally obscured by the HFEA's headline figures. Firstly, our analysis gives four clear and distinct reasons why patients might be freeze their eggs. Secondly, we show that while women who freeze their eggs for 'social' reasons are predominantly single, patients whose eggs are frozen for clinical or incidental reasons are most likely to be in heterosexual relationships. And thirdly, by showing that a large majority of the thawed eggs in our sample come from CEF and IEF cycles, we provide a clear explanation for why the national figures from 2016 show that, "Of the thawed egg cycles performed, well over half used eggs which had been frozen for less than a year" (HFEA, 2016: 27). 
Indeed, the differences with regards to the current status of eggs between the different categories of egg freezing are possibly the most striking findings of this analysis. If we look at each of the two largest categories in more detail, we can see that while almost all of the eggs from SEF cycles (92.8\%) between 2012-2016 are still in storage, almost all of the eggs from CEF cycles (90.2\%) have already been thawed to attempt conception. These distinct differences should make us rethink our assumptions and require us to conduct further detailed analysis in order to provide a better understanding of subsequent success rates or pregnancy outcomes for egg freezing patients from different categories.

\section{Limits of the current study}

This paper has some limitations that impact how the data can be used and understood. Firstly, it must be noted once again that this paper presents analysis pertaining to records from one clinic only, the LWC in Harley Street, and cannot therefore be generalised. Whilst the LWC is one of the clinics providing the highest number of egg freezing cycles in the UK, it is fair to assume that no single clinic can be truly representative of national patterns. Indeed, there are some important differences between our data and the HFEA's national figures with regards to, for example, the age distribution and relationship status of egg freezing patients. In fact, it is safe to assume that the proportion of the different categories we have identified will be varied among different clinics providing egg freezing in the UK. For example, some NHS IVF clinics will be providing a much higher proportion of medical egg freezing cycles, and the freezing and 'batching' of eggs in CEF cycles will be practiced by some clinics and not others. Furthermore, since the LWC Harley Street is a Londonbased clinic with a higher than average proportion of single women patients, it is possible that some of the year-on-year increase in the number of egg freezing cycles can be explained by the LWC capturing a larger proportion of the UK egg freezing market rather than reflecting the real rate of increase of the practice nationally, though it is difficult to be exact about this because of the discrepancies in how an egg freezing cycle was defined in the two HFEA reports of 2016 and 2018 (for a discussion of this see, Gurtin, 2018).

Secondly, the current study may be criticised for being too UK-focused. This is indeed correct, but we would argue that the strength of the analysis comes from its specificity and detail, and therefore the possibilities provided to look behind broader headline figures. Internationally speaking, there will no doubt be important differences in the practice of egg freezing, including its legality, popularity, and patterns of use. For example, there may be other categories of egg freezing (such as, for example, Ethical Egg Freezing) that are not present in our data set but may prove important in other national contexts. Similarly, it would be fair to expect that the demographic characteristics of egg freezing patients will vary and show different patterns depending on the particularities of different national and regulatory contexts. For example, the average age of egg freezing patients in the UK is likely to be higher than the average age of egg freezing patients in the US, influenced not only by the different cultural contexts in the two countries, but also directly by the HFEA's restrictive 10-year storage limit for frozen eggs. However, despite national specificities, the data presented is 
nevertheless extremely useful in alerting us to the heterogeneity of egg freezing patients and categories and should encourage other clinics, and national bodies collecting fertility treatment data, to examine the constitution of their own egg freezing patient populations.

A third limitation of this study is that it makes use of existing records to create a quantitative and numerical analysis, and does not provide qualitative details regarding women's own opinions and experiences of egg freezing. Without doubt, it would be extremely valuable to elicit women's own views regarding their reasons for egg freezing and to receive their responses to the categories presented here. To this end, some of the researchers of the current paper are currently engaged in qualitative studies of women's experiences of egg freezing and we hope to report from these in due course.

Even taking these limitations into account, the analysis presented here provides a strong case for the hitherto unrecognised heterogeneity of the egg freezing practices and the importance of recognising different categories of egg freezing. This leads us to make some recommendations regarding clinical and national data collection on egg freezing and regulation in the future.

\section{Ethical and regulatory implications}

The current HFEA data should be understood as comprising a mixture of the four categories presented in this paper, and thus the picture with regards to any one category may be blurred or even obscured by data conflation. For example, it is likely that many of the children born from frozen-thawed eggs are not actually born to 'social egg freezers' but to freezers from other categories (in particular, CEF and IEF). As such, the real number of children born to women who have deliberately postponed motherhood by freezing their eggs is likely to be far lower even than the very low figures provided by the HFEA, which stated that 60 children had been born from thawed eggs by 2013. This is important because it suggests that we need more detailed analysis at the national level, not only in order to understand the relative outcomes or success rates for egg freezing patients from different groups, but also to provide more accurate information to potential users. Such data would also help to create clinical and ethical parameters to ensure that egg freezing is practiced "for better, not worse" (Mertes and Pennings, 2011).

Future data collection, including by the HFEA and by clinics, should record details about the reason for egg freezing in order to provide clearer information regarding both the trajectories and outcomes of eggs frozen for different reasons by different patient groups. We would recommend that the categories identified in this paper become standard in the collection and analysis of future egg freezing data, and that other clinics or countries supplement these categories as required by different patient populations. For example, if substantial numbers of women report freezing eggs for ethical reasons in a given country, Ethical Egg Freezing (EEF) could become an additional category for use in data collection and analysis. 
We argue that, dividing the different categories of egg freezing will help women considering this procedure to gain a much more accurate and relevant understanding of egg freezing as it applies to their situation, including its prevalence among women like themselves and its success rates as judged by subsequent pregnancies and live births among such a group. From an ethical perspective, this is important for enabling potential patients to make sound, empirically-based decisions about whether or not (or when) to freeze their eggs, and to be able to give truly informed consent to such complex treatments (see also Jackson, 2017).

\section{Future research}

As one of the most interesting aspects of the current analysis was the differential rate of thawing among egg freezing patients from different categories, we have also undertaken an analysis of ten years of egg thaw data at the LWC, which we will be publishing separately. This analysis will provide much greater detail on the demographic characteristics of patients thawing their eggs, including comparisons between age and relationship status at time of freeze and time of thaw for patients from each of the different freezing categories identified here. Since the great majority of SEF patients still had their eggs in storage, it would be extremely interesting to find out more about the intentions and opinions of these women. In the UK, frozen eggs can be stored for a maximum of ten years and it would be particularly important to elicit the views and opinions of women nearing their storage limit (see Jackson, 2016).

Although the current paper reports on a retrospective and quantitative analysis, we recognise that it is extremely important that the women who make up the figures and data we report on have a chance to tell their own stories and have their voices heard. As such, the lead author is conducting a range of interviews with women who have frozen their eggs, and in some cases have already come back to thaw them to attempt conception, and is committed to presenting their perspectives as evidenced through such qualitative research. Egg freezing is still a new technology, with its social impact yet to be understood; and, ultimately, it is the thoughts, feelings and experiences of its "social pioneers" - the early cohort of women who have decided to freeze their eggs - that will prove the most illuminating and meaningful.

\section{ACKNOWLEDGEMENTS}

\section{Acknowledgements}

The authors gratefully acknowledge the clinical work undertaken by a large team of clinicians, nurses and embryologists that has enabled these data analysis to occur, and we appreciate the suggestions and comments made by Professor Susan Golombok on earlier drafts. 


\section{Declaration of interests and funding}

There is no financial or commercial conflict of interest.

The authors work in the clinic from which the data was obtained.

There was no independent funding received for the study.

\section{Contribution of each author}

ZG - Designed concept and analysis; collected and analysed data; generated concepts; wrote paper.

TS - Helped with initial stage of data collection.

JW - Provided access to data, as head of lab from which data was gathered.

KA - Helped in concept design; provided overview; head of clinic from which data was gathered.

\section{REFERENCES}

American Society of Reproductive Medicine, Society for Assistive Reproductive Technology (2013) Mature oocyte cryopreservation; a guideline. Fertility and Sterility, 99(1): 37-43. Published online Oct 22, 2012.

Argyle, C.E., Harper, J.C. and Davies, M.C. (2016) Oocyte cryopreservation: where are we now? Human Reproduction Update 22(4): 440-449.

Baldwin, K., Culley, L., Hudson, N. and Mitchell, H. (2014) Reproductive technology and the life course; Current debates and research in social egg freezing. Human Fertility, 17(3): 170-179.

Baldwin, K., Culley, L., Hudson, N., Mitchell, H. and Lavery, S. (2015) Oocyte cryopreservation for social reasons: demographic profile and disposal intentions of UK users. RBMOnline, 31: 239-245.

Belluck, P. (2018) What Fertility Patients Should Know About Egg Freezing, The New York Times, 13 March 2018.

Browne, J. (2018) Technology, Fertility and Public Policy: A Structural Perspective on Human Egg Freezing and Gender Equality. Social Politics Fall, 1-20.

Chen, C. (1986) Pregnancy after human oocyte cryopreservation. Lancet, 1: 884-886.

Cobo A., Kuwayama M., Perez S., Ruiz A., Pellicer A., Remohi J. (2008) Comparison of concomitant outcome achieved with fresh and cryopreserved donor oocytes vitrified by the Cryotop method. Fertil. Steril. 89: 1657-1664.

Cobo, A., Meseguer M., Remohi J, Pellicer A. (2010) Use of cryo-banked oocytes in an ovum donation programme: a prospective randomized, controlled, clinical trial. Hum Reprod, 25: 2239-2246.

Dondorp, W., de Wert, G., Pennings, G., Shenfield, F., Devroey, P., Tarlatzis, B., Barri, P., Diedrich, K. and ESHRE Task Force on Ethics and Law (2012) Oocyte cryopreservation for age-related fertility loss. Human Reproduction, 27(5): 1231-1237. 
Gurtin, Z.B. (2018) Unscrambling HFEA data on egg freezing: where are the missing frozen eggs? BioNews, 943. https://www.bionews.org.uk/page 135010

HFEA (2016) Fertility treatment 2014 - Trends and figures. Human Fertilisation and Embryology Authority. https://www.hfea.gov.uk/media/1783/fertility-treatment-2014-trends-and-figures.pdf

HFEA (2018) Fertility treatment 2014-2018 - Trends and figures. Human Fertilisation and Embryology Authority. https://www.hfea.gov.uk/media/2544/hfea-fertility-treatment-2014-2016trends-and-figures.pdf

Jackson, E. (2016) 'Social' egg freezing and the UK's statutory storage time limits. J Med Ethics, 0: 14.

Jackson, E. (2017). The ambiguities of 'social' egg freezing and the challenges of informed consent. BioSocieties, 1 - 20.

Mertes, H. and Pennings, G. (2011) Social egg freezing: for better, not worse. RBMOnline, 23(7): 824829.

Parmegiani L, Cognigni GE, Bernardi S, Cuomo S, Ciampaglia W, Infante FE, Tabarelli de Fatic C, Arone A, Maccarini AM, Filicon M. (2011) Efficinetcy of aspetic open vitrification and hermetical cryostorage of human oocytes. Reprod Biomed Online, 23: 505-5012.

Rienzi L, Romano S, Albricci L, Maggiuli R, Capalbo A, Baroni E, Colamaria S, Sapienza F, Ubaldi F. (2010) Embryo development of fresh 'versus' vitrified metaphase II oocytes after ICSI: a prospective randomized sibling-oocyte study. Hum Reprod, 24: 66-73.

Specter, F. (2017). Hope or hype? The chilling truth about freezing your eggs. The Guardian. https://www.theguardian.com/lifeandstyle/2017/nov/06/hope-or-hype-the-chilling-truth-aboutfreezing-your-eggs

Wise, J. (2000) UK lifts ban on frozen eggs. 320:334.

Wooller, S. (2017). No Good Eggs: lack of 'high quality' men is the reason women are freezing their eggs to preserve their fertility, experts say. The Sun.

https://www.thesun.co.uk/living/3948792/women-are-freezing-their-eggs-in-a-bid-to-preservefertility-due-to-not-enough-high-quality-men-a-study-suggests/ 